\title{
Recent results for UPC at the LHC
}

\author{
Mariola Kłusek-Gawenda ${ }^{1,3, *}$ \\ ${ }^{1}$ Institute of Nuclear Physics Polish Academy of Sciences, PL-31342 Krakow, Poland
}

\begin{abstract}
Ultraperipheral heavy ion collisions are a source of photons which can collide with each other producing a pair of particles. This work will be focused on analysis of the light-by-light scattering. Here contribution from fermionic boxes, resonance scattering, VDM-Regge model, two-gluon exchange and pionic background will be compared. Each of these processes dominate at different ranges of two-photon invariant masses. Our calculated nuclear cross section is in good agreement with recently measured ATLAS and CMS data. Predictions including ALICE and LHCb experimental cuts for the next run at the LHC will be shown.
\end{abstract}

\section{Introduction}

Physics of the ultraperipheral collisions (UPC) of heavy ions gives a nice opportunity to study electromagnetic processes. Due to the very strong electromagnetic field of colliding nuclei, reactions relate to photon collisions can be studied. One can consider $\gamma \gamma$ fusion and photoproduction (Pomeron and/or Reggeon exchange) as a sub-process of heavy ion UPC. This proceedings will pertain to light-by-light scattering. Diphoton processes have long been studied at $e^{+} e^{-}$collider. This tool allows to test a QED theory and a lot of aspects of meson spectroscopy. The first theory concerning the possibility of the light-by-light scattering was proposed more than 80 years ago i.a. by W. Heisenberg and his students : H. Euler and B. Kockel [1, 2] or by A. Akhieser, L. Landau and I. Pomeranchuk [3].

Section 2 includes discussion of a elementary cross section for a given type of $\gamma \gamma \rightarrow \gamma \gamma$ sub-process. In the next section we present theoretical approach which is used to calculate the nuclear cross section. Section 4 contains a comparison of our results with existing experimental data and some predictions for the next run of the LHC.

\section{Elementary cross section}

The leading order of elementary cross section for $\gamma \gamma \rightarrow \gamma \gamma$ process is well-know and one can use an available to the general public Mathematica package: FormCalc [4]. The (a) diagram in Fig. 1 shows so-called fermionic box (scattering via quarks and leptons is taken into account). The next diagram presents $W^{+} W^{-}$boson loop and this cross section is calculated within LoopTools [5]. Fig. 1(c) presents diagram for non-perturbative mechanism of both photons fluctuation into vector mesons and their subsequent interaction. This involves the Reggeon and Pomeron exchanges between $\rho, \omega$ or $\phi$ light mesons. The last diagram of Fig. 1 is the same order in $\alpha_{e m}$ as previous one but has higher order in $\alpha_{s}$. The two-gluon exchange

\footnotetext{
*e-mail: mariola.klusek@ifj.edu.pl
} 
(a)

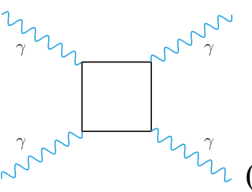

(b)

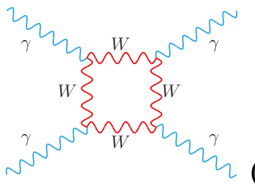

(c)

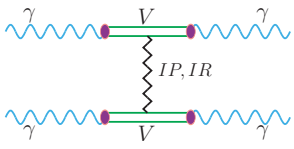

(d)

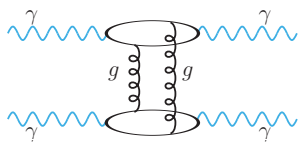

Figure 1. $\gamma \gamma \rightarrow \gamma \gamma$ scattering: (a) fermionic boxes, (b) one-loop $W$ box, (c) the VDM-Regge, (d) two-gluon exchange mechanism.

mechanism is a three-loop mechanism [6]. The finite fermion masses, the full momentum structure in the loops and all helicity amplitudes are included. Considering processes with gluon exchange, one can use so-called regularization parameter, $m_{g}$, which can be: $m_{g}=0$ for usual gluon exchange, however $m_{g}=0.75 \mathrm{GeV}$ is suggested by lattice QCD [7].

(a)

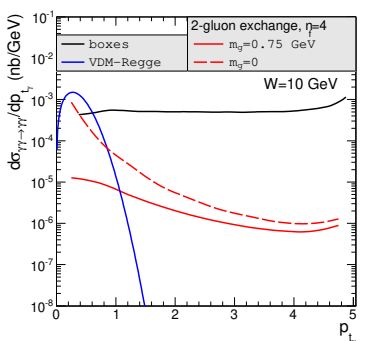

(b)

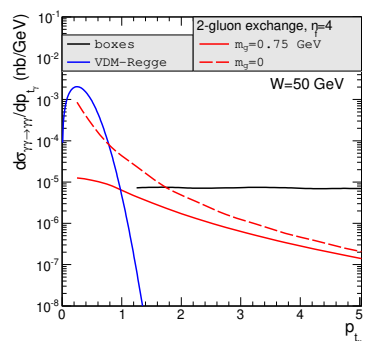

(c)

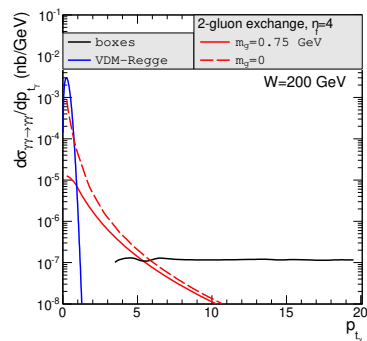

Figure 2. The differential cross section as a function of transverse momentum of one of two outgoing photons for $\gamma \gamma \rightarrow \gamma \gamma$ scattering. Contribution of fermionic boxes (black lines), VDM-Regge (blue lines) and two-gluon exchange (red lines) are presented. Here energy $W=\sqrt{\hat{s}}$ is: (a) $10 \mathrm{GeV}$, (b) $50 \mathrm{GeV}$ and (c) $200 \mathrm{GeV}$.

With the help of Fig. 2 we present the importance of each mechanism at fixed value of energy ((a) $-W=10 \mathrm{GeV}$, (b) $-W=50 \mathrm{GeV}$, (c) $-W=200 \mathrm{GeV})$. The soft VDMRegge contribution plays important role only at the region of very small $p_{t}$. Above about $1 \mathrm{GeV}$ the two-gluon exchange contribution is larger than the VDM-Regge component. Only at $W=200 \mathrm{GeV}$ there is some window of photon transverse momenta $p_{t}=(1-5) \mathrm{GeV}$ where two-gluon contribution should be experimentally observed. The gluon mass has a large effect on the cross section in a broad range of $p_{t}$. The convergence of the result with massless gluon and that with $m_{g}=0.75 \mathrm{GeV}$ appears only at relatively large $p_{t} \approx 5 \mathrm{GeV}$. The box contribution extend over broad range of $p_{t}$ and dominates at smaller energy. For larger energy, box diagrams cannot be uncredited to $\gamma \gamma \rightarrow \gamma \gamma$. This elastic scattering could be studied experimentally in a future photon-photon collider. This will be realized at the facilities such as the International $e^{+} e^{-}$Linear Collider.

In Ref. [8] the role of mesons exchanges in light-by-light scattering was studied. There several pseudoscalar, scalar, tensor and 4-spin mesons were taken into account. The authors considered not only $s$-channel but also for the first time $t$ - and $u$-channel meson exchange amplitudes corrected for off-shell effects including vertex form factors. Formally, the angular distribution for the $s$-channel resonances is typically used in calculating Feynman diagram contributions in the form:

$$
\frac{\mathrm{d} \sigma(\gamma \gamma \rightarrow R \rightarrow \gamma \gamma)}{\mathrm{d} z}=\frac{1}{32 \pi W_{\gamma \gamma}^{2}} \frac{1}{4} \sum_{\lambda_{1}, \lambda_{2}}\left|\mathcal{M}_{\gamma \gamma \rightarrow R \rightarrow \gamma \gamma}\left(\lambda_{1}, \lambda_{2}\right)\right|^{2},
$$


where $z=\cos \theta$ and $\theta$ denotes the angle between the beam direction and the outgoing particle in the c.m. frame, $W_{\gamma \gamma}$ is the invariant mass of the $\gamma \gamma$ system. The amplitude for the $\gamma \gamma$ production through the $s$-channel exchange of a pseudoscalar/scalar mesons is written as

$$
\mathcal{M}\left(\lambda_{1}, \lambda_{2}\right)=\frac{\sqrt{64 \pi^{2} W_{\gamma \gamma}^{2} \Gamma_{R}^{2} B r^{2}(R \rightarrow \gamma \gamma)}}{\hat{s}-m_{R}^{2}-i m_{R} \Gamma_{R}} \times \frac{1}{\sqrt{2 \pi}} \delta_{\lambda_{1}-\lambda_{2}} .
$$

Here we use the same notation as in Ref. [9]. In the present analysis we take into account only pseudoscalar and scalar mesons: $\eta, \eta^{\prime}(958), \eta_{c}(1 S), \eta_{c}(2 S), \chi_{c 0}(1 P)$. Their masses $m_{R}$, total widths $\Gamma_{R}$ and branching ratios $\operatorname{Br}(R \rightarrow \gamma \gamma)$ are taken from PDG [10].

In addition, also background from the $\gamma \gamma \rightarrow \pi^{0}(\rightarrow \gamma \gamma) \pi^{0}(\rightarrow \gamma \gamma)$ process is considered. In Ref.[9] we constructed a multi-component model which described e.g. the Belle [11] and Crystal Ball [12] data for $\gamma \gamma \rightarrow \pi^{0} \pi^{0}$. In [9] both $\gamma \gamma \rightarrow \pi^{+} \pi^{-}$and $\gamma \gamma \rightarrow \pi^{0} \pi^{0}$ reactions were considered within a multi-component model. There, for the first time, both the total cross section and angular distributions and significance of nine resonances, $\gamma \gamma \rightarrow \pi^{+} \pi^{-} \rightarrow \rho^{ \pm} \rightarrow \pi^{0} \pi^{0}$ continuum, Brodsky-Lepage and handbag mechanisms in these processes was studied. A detailed formalism and the description of these sub-processes can be found in [9]. The angular distribution for the $\gamma \gamma \rightarrow \pi^{0} \pi^{0}$ process in nuclear collisions can be calculated similarly as in Eq. (1). Then $\theta$ is the pion scattering angle. If only two photons from different neutral pions are measured at a given experimental range of rapidities and transverse momenta such an event could be wrongly identified as $\gamma \gamma \rightarrow \gamma \gamma$ scattering if no extra cuts are imposed to reduce or eliminate such a background.

\section{Nuclear cross section}

The nuclear cross section is calculated with the help of equivalent photon approximation (EPA) [13-15]. This theory uses concept of photon fluxes which depend on energy of photon $\left(\omega_{i}\right)$ and impact parameter $\left(\mathbf{b}_{i}\right)$. Here we consider only ultraperipheral collisions that is impact parameter $(b)$ determining distance between medium of colliding nuclei is bigger than the sum of the radii. Fig. 3(a) reflects quantities which are used in the impact parameter space. The end of $\mathbf{b}_{i}$ vectors set a position where the pair of particles is produced. Here we mean just two-photon production. A schematic diagram illustrating process of heavy ion collision that is associated with production of some final state $X$ is presented in Fig.3(b). It is often used rather photon number $\left(n\left(\omega_{i}\right)\right)$ instead of photon flux $\left(N\left(\omega_{i}, \mathbf{b}_{i}\right)\right)$ (where $i=1,2$ numbers nuclei) but we think that usage of EPA in the impact parameter space is more accurate. To calculate nuclear cross section for $A A \rightarrow A A X$ process one needs to know the probability that first nucleus emits a photon with the energy $\omega_{1}$ and, simultaneously, second nucleus emits a photon with the energy $\omega_{2}$. Next, they collide with each other to produce some final state $X$ (here it is $\gamma \gamma$ or $\pi^{0} \pi^{0}$ state).

In our approach, we use equivalent photon approximation in the impact parameter space. The cross section is then the 5-dimensional integral:

$$
\begin{aligned}
\sigma_{A_{1} A_{2} \rightarrow A_{1} A_{2} X_{1} X_{2}}\left(\sqrt{s_{A_{1} A_{2}}}\right) & =\int \sigma_{\gamma \gamma \rightarrow X_{1} X_{2}}\left(W_{\gamma \gamma}\right) N\left(\omega_{1}, \mathbf{b}_{1}\right) N\left(\omega_{2}, \mathbf{b}_{2}\right) S_{a b s}^{2}(\mathbf{b}) \\
& \times \mathrm{d}^{2} b \mathrm{~d} \bar{b}_{x} \mathrm{~d} \bar{b}_{y} \frac{W_{\gamma \gamma}}{2} \mathrm{~d} W_{\gamma \gamma} \mathrm{d} Y_{X_{1} X_{2}},
\end{aligned}
$$

where $X_{1} X_{2}$ is a pair of produced particles, $\bar{b}_{x}$ and $\bar{b}_{y}$ are components of $\mathbf{b}_{i}$ vector, the energy of produced particles depends on energy of photons as follows: $W_{X_{1} X_{2}}=2 \sqrt{\omega_{1} \omega_{2}}$ and rapidity of outgoing system is expressed through rapidity of singles particles: $Y_{X_{1} X_{2}}=\frac{1}{2}\left(y_{X_{1}}+y_{X_{2}}\right)$. 
(a)

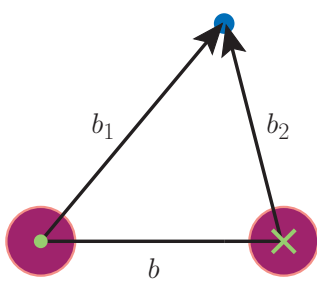

(b)

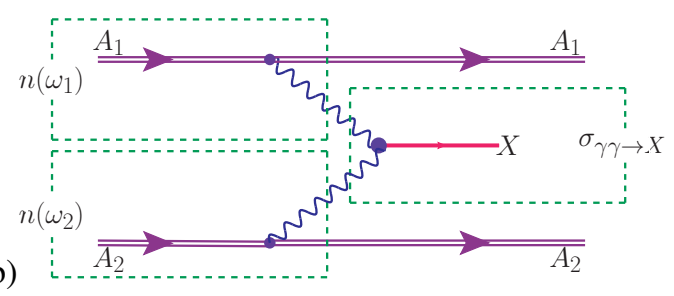

Figure 3. (a) A view perpendicular to the direction of two ion motion. (b) Feynman diagram for $\mathrm{AA} \rightarrow \mathrm{AA} X$ process.

Absorption factor $S_{a b s}(\mathbf{b})$ assures UPC which means that the nuclei do not undergo nuclear breakup. Very often we extend Eq. 3 by additional dimension. This allows to make a more accurate prediction or description of experimental data. For example, we replace the total elementary cross section $\sigma_{\gamma \gamma \rightarrow X_{1} X_{2}}$ by angular distribution. Then one has $\mathrm{d} \sigma_{A_{1} A_{2} \rightarrow A_{1} A_{2} X_{1} X_{2}}\left(\sqrt{s_{A_{1} A_{2}}}\right) / \mathrm{d} y_{X_{1}} \mathrm{~d} y_{X_{2}} \mathrm{~d} p_{t}$ multi-dimensional distribution. Such a grid is prepared with the help of Monte Carlo simulation using the VEGAS Algorithm [16].

(a)

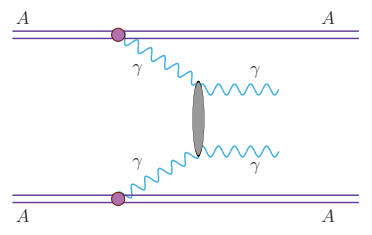

(b)

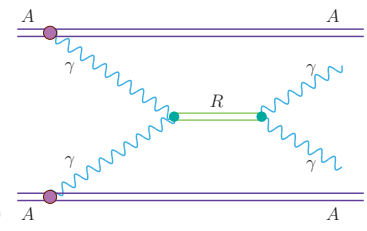

(c)

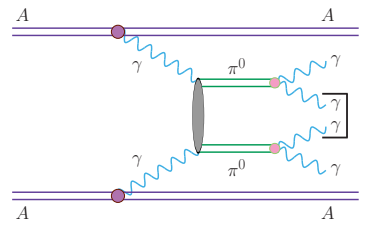

Figure 4. Diagrams for nuclear $A A \rightarrow A A \gamma \gamma$ mechanism. Different subprocesses can be signalized: (a) Standard Model box (b) resonances (c) pionic background.

A current analysis concerns the production of diphoton pairs originating from

- boxes, VDM-Regge mechanism and two-gluon exchange (see Fig. 4(a)),

- resonances which decay into $\gamma \gamma$ state (see Fig. 4(b)),

- pionic background (see Fig. 4(b)).

Eq. (3) allows to calculate nuclear cross section for each of bulleted processes.

The flux of photons, especially at small $b$, very strongly depends on the nuclear form factor. We prefer to use a realistic form factor in our calculation. Then nucleus is treated as a object with realistic charges distribution. Thereby the Wood-Saxon density profile is included. Often a monopole form factor is used in the calculation. The comparison of results that include realistic and monopole form factor will be presented below. Nuclear form factor kills large virtualities in UPC of heavy ion, therefore, the initial photon virtualities equal to almost zero $\left(Q_{1}^{2}, Q_{2}^{2} \approx 0\right)$.

\section{Comparison with experimental data and predictions for future experiments}

Light-by-light scattering was realized experimentally only recently $[17,18]$. About one year before the first experimental results which were obtained by the ATLAS group, we have studied light-by-light scattering via fermion loop and with the help of non-perturbative mechanism of photons fluctuation into light vector mesons (VDM-Regge model) [19]. We have 
considered only ultraperipheral lead-lead collisions. For ions of charges $Z_{1}, Z_{2}$, the cross section is enhanced by $Z_{1}^{2} Z_{2}^{2}$ factor compared to proton-proton collisions, at least at low diphoton invariant masses equal to diphoton collision energies, where the initial photons are quasi real with extremely low virtualities. But on the other hand, a significant part of cross section is cut by absorption factor (Eq. (3)) which ensures ultraperipheral character of the process.

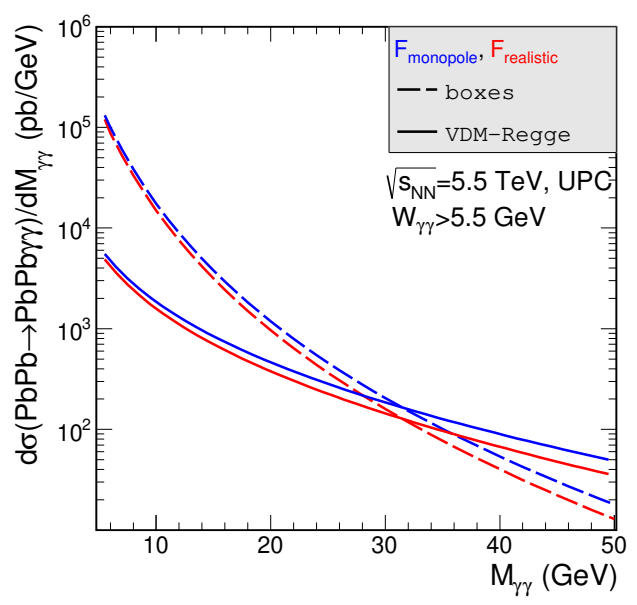

Figure 5. Differentail cross section as a function of diphoton invariant mass. Blue lines - nuclear calculations include the monopole form factor. Red lines - results with the realistic form factor. Solid lines - boxes contribution and dashed lines relates to VDM-Regge model.

Fig. 5 illustrates a comparison of contributions of boxes (dashed lines) and VMDRegge mechanism (solid lines) in nuclear calculations taking into account the monopole (blue lines) and realistic form factor (red lines). One can see a differential cross section for the $\mathrm{PbPb} \rightarrow \mathrm{PbPb} \gamma \gamma$ reaction in heavy ion UPC at $\sqrt{s_{N N}}=5.5 \mathrm{TeV}$ with extra cut on $W_{\gamma \gamma}>5.5 \mathrm{GeV}$. The cross section obtained with the monopole form factor is more than $10 \%$ bigger than that obtained with the form factor which is calculated as a Fourier transform of the charge distribution in the nucleus. The ratio between those two results become larger with larger value of the diphoton invariant mass. The competition of the two mechanisms seems to be interesting. While at low energies the box contribution wins, at $M_{\gamma \gamma}>30 \mathrm{GeV}$ the VDM-Regge contribution is bigger.

ATLAS measured a fiducial cross section of $\sigma=70 \pm 24$ (stat.) \pm 17 (syst.) nb [17] and our theoretical calculations (including experimental acceptance) gave $49 \pm 10 \mathrm{nb}$ [19]. The ATLAS comparison of its experimental results to the predictions from Ref.[19] show a reasonable agreement (see Fig. 3 in Ref. [17]). Only 13 events (7.3 signal events and 2.6 background events) were observed by the ATLAS collaboration. This detector recorded data using $480 \mu \mathrm{b}^{-1}$ of lead-lead collision at the centre-of-mass energy per nucleon pair of 5.02 $\mathrm{TeV}$. Measurement of diphoton pair was done in midrapidity region. The $\gamma \gamma$ invariant mass was limited to $M_{\gamma \gamma}>6 \mathrm{GeV}$. Similarly as the ATLAS collaboration, the CMS group measured the same process but for somewhat lower threshold of invariant mass of the produced diphotons [18]. The measured fiducial light-by-light scattering cross section, $\sigma=120 \pm 46$ (stat.) \pm 28 (syst.) \pm 4 (theo.) $\mathrm{nb}$. We have recalculated this process including the CMS acceptance and we obtained $\sigma=(103 \pm 0.034) \mathrm{nb}$ which is in a good agreement with the CMS result. Fig. 6 shows the CMS preliminary experimental data (red points) together with 
our theoretical histogram (blue area). The panel (a) depicts diphoton invariant mass and the panel (b) corresponds to rapidity distribution of single outgoing photon. One can observe rather large statistical uncertainties. Our calculations agree give agreement with data but it seems to be important to further test the light-by-light scattering with a better precision.

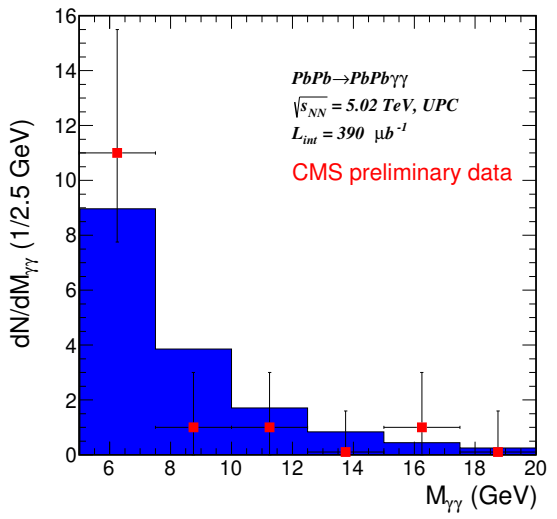

(a)

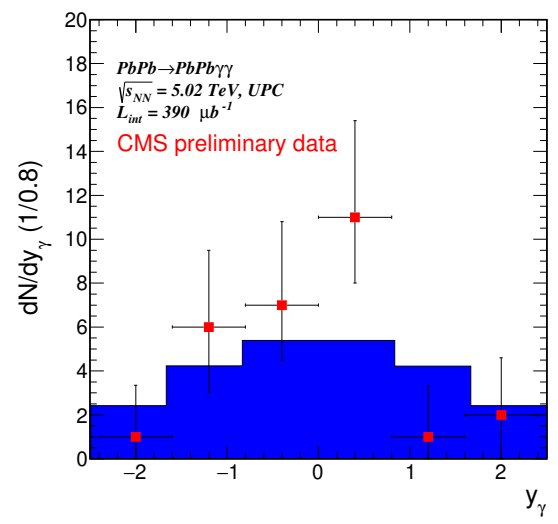

(b)

Figure 6. Comparison of our results with existing CMS preliminary data [18]. (a) Distribution in invariant mass of diphoton pair. (b) Rapidity of single outgoing photon.

Due to relatively large cuts on the photon transverse momenta, only relatively large diphoton invariant masses were measured by the ATLAS and CMS collaborations. We believe that in a future one could go to larger luminosity, higher collision energies, better statistics and smaller diphoton invariant masses.

Table 1. Fiducial region used in the analysis.

\begin{tabular}{ccc}
\hline experiment & pseudorapidity range & other condition \\
\hline ALICE & $-0.9<\eta_{\gamma}<0.9$ & $E_{\gamma}>200 \mathrm{MeV}$ \\
LHCb & $2.0<\eta_{\gamma}<4.5$ & $p_{t, \gamma}>200 \mathrm{MeV}$ \\
\hline
\end{tabular}

Next, predictions for the ALICE and LHCb experiment will be shown. Table 1 presents details of the ALICE and LHCb (semi)acceptance which is included in the calculation. The ALICE detector facilitates a measurement of outgoing photons at midrapidity region [20]. Photons with transverse energy smaller than $200 \mathrm{MeV}$ cannot be detected. The above calculations will be compared with the results for more forward rapidity region: $2<\eta<4.5$ [21]. Here we assume that any photon with $p_{t, \gamma}>200 \mathrm{MeV}$ will be measured.

Fig. 7 illustrates contribution of $\gamma \gamma \rightarrow \gamma \gamma$ signal (black line), background (blue dashed line) and resonances (green lines) to the diphoton invariant mass distribution for ALICE (a) and LHCb conditions (b). The background is composed of events where exactly two of four outgoing photons are detected. The first one comes from the first pion, and the second one comes from the second pion. The two other photons, from the $\pi^{0} \pi^{0} \rightarrow(\gamma \gamma)(\gamma \gamma)$ decays, are then outside of detection area. Contributions suggest that one could be able to measure the LO QED fermionic signal above $M_{\gamma \gamma}>2 \mathrm{GeV}$. Below this value, two very clear peaks show up, corresponding to $\eta$ and $\eta^{\prime}(958)$ mesons that decay into two-photon final state. Inclusion of the Gaussian distribution to simulate experimental energy resolution causes only a little smearing of the resonant signals. Then the transverse momenta of each of the photons takes 


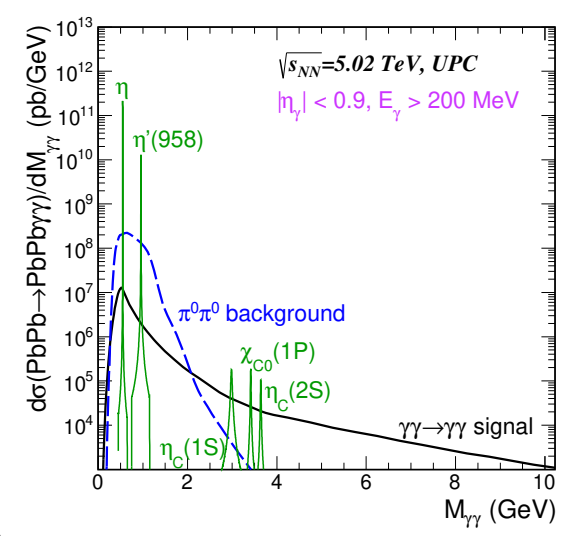

(a)

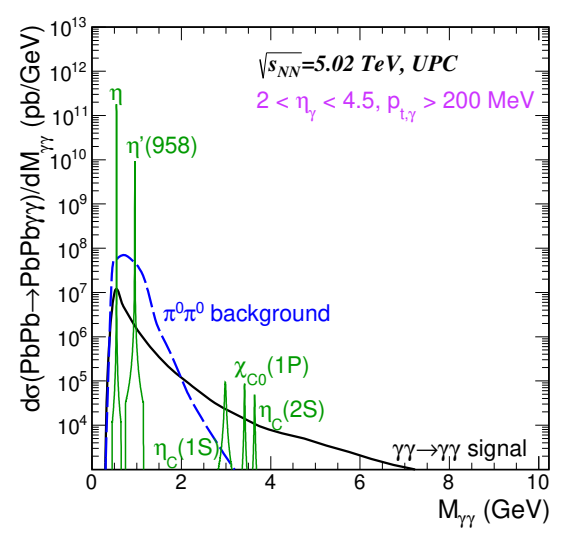

(b)

Figure 7. Differential nuclear cross section for $\mathrm{PbPb} \rightarrow \mathrm{PbPb \gamma \gamma}$ as a function of $\gamma \gamma$ invariant mass. (a) ALICE and (b) LHCb kinematical cuts are included. In addition, the energy experimental resolution is taken into account. Here $\sqrt{s_{N N}}=5.02 \mathrm{TeV}$.

the form: $p_{i, t}=p_{t}+\left(\frac{p_{t}}{E_{i}}\right) \delta E_{i}$. It seems to worth mentioning that the peak corresponding to a resonance very strongly depends on the number of bins. The maximum value of the differential cross section emerges exactly at $m_{R}$.

Table 2. Total nuclear cross section in nb.

\begin{tabular}{l|rr|rr}
\hline Energy & \multicolumn{2}{|c|}{$W_{\gamma \gamma}=(0-2) \mathrm{GeV}$} & \multicolumn{2}{c}{$W_{\gamma \gamma}>2 \mathrm{GeV}$} \\
\hline Fiducial region & ALICE & LHCb & ALICE & LHCb \\
\hline boxes & 4890 & 3818 & 146 & 79 \\
$\pi^{0} \pi^{0}$ background & 135300 & 40866 & 46 & 24 \\
$\eta$ & 722573 & 568499 & & \\
$\eta^{\prime}(958)$ & 54241 & 40482 & & \\
$\eta_{c}(1 S)$ & & & 9 & 5 \\
$\chi_{c 0}(1 P)$ & & & 4 & 2 \\
$\eta_{c}(2 S)$ & & & 2 & 1 \\
\hline
\end{tabular}

In Table 2 one can find values of the total nuclear cross section for fermionic boxes, pionic background and five types of mesons. Cross section is given in two ranges of the diphoton invariant masses. The first one is from 0 to $2 \mathrm{GeV}$ and the second one from $2 \mathrm{GeV}$ to $50 \mathrm{GeV}$. Here a cut on pseudorapidity and energy or transverse momentum of photons is included. As mentioned, the effect of energy resolution has a major impact on smearing of mesonic contribution and does not change the value of the cross section. The largest cross section is obtained for the $\gamma \gamma \rightarrow \eta \rightarrow \gamma \gamma$ resonance scattering. Additionally, in the range of diphoton invariant mass $M_{\gamma \gamma}>2 \mathrm{GeV}$, comparison of cross sections for fermionic boxes and pionic background clearly shows almost fourfold dominance of boxes over non-wanted background.

Fig. 8 corresponds to the next run at the LHC. The energy (per nucleon) of heavy-ion collision was calculated as follows:

$$
\sqrt{s_{N N}}=\sqrt{\frac{Z_{1} Z_{2}}{A_{1} A_{2}}} \sqrt{s_{p p}}
$$




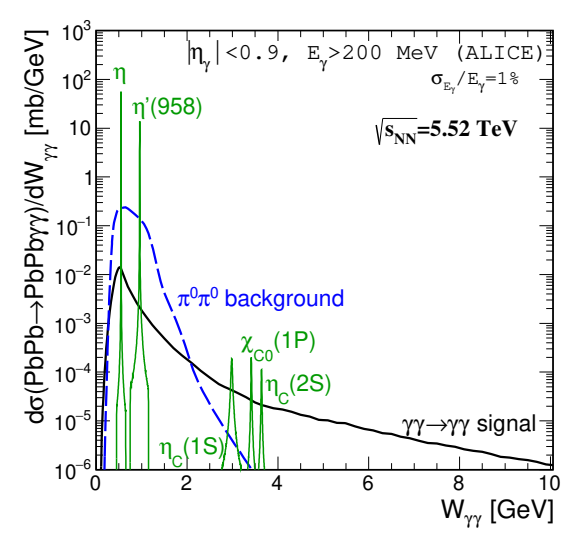

(a)

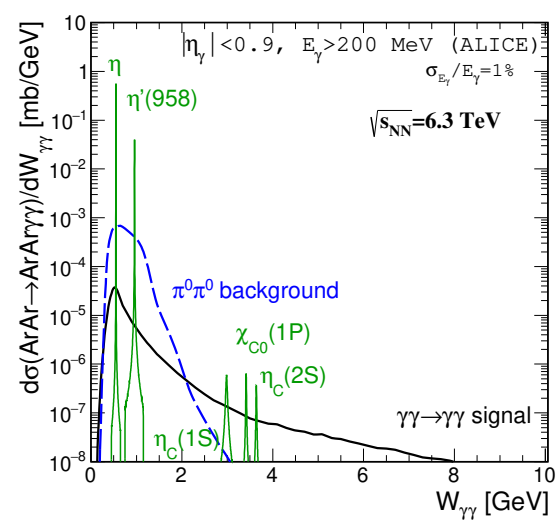

(b)

Figure 8. Differential cross section as a function of $W_{\gamma \gamma}=M_{\gamma \gamma}$ for (a) $\mathrm{PbPb} \rightarrow \mathrm{PbPb} \gamma \gamma$ and (b) $\operatorname{ArAr} \rightarrow \operatorname{ArAr} \gamma \gamma$. The collision energy at the center-of-mass of the heavy ion collision is $5.52 \mathrm{TeV}$ and $6.3 \mathrm{TeV}$ for lead-lead and argon-argon respectively.

for the energy in the center-of-mass of proton-proton collision $\sqrt{s_{p p}}=14 \mathrm{TeV}$. Then predicted values for ${ }^{208} \mathrm{~Pb}^{82+}{ }_{2}^{208} \mathrm{~Pb}^{82+}$ collision is $\sqrt{s_{N N}}=5.52 \mathrm{TeV}$ (Fig. 8(a)) and for ${ }^{40} \mathrm{Ar}^{18+}$ ${ }^{40} \mathrm{Ar}^{18+}$ it is $\sqrt{s_{N N}}=6.3 \mathrm{TeV}$ (Fig. 8(b)). The analysis focuses on lower diphoton invariant masses again. At lower energies $\left(W_{\gamma \gamma}<4 \mathrm{GeV}\right)$ meson resonances may play important role in addition to the Standard Model box diagrams or proposed pionic background. The inclusion of energy resolution has a significance mainly at $\gamma \gamma \rightarrow \eta, \eta^{\prime} \rightarrow \gamma \gamma$ resonance scattering and this contribution will be measured with a good statistics. However, the resonance signal is modified including experimental energy resolution [22] and the $\eta$ and $\eta^{\prime}(958)$ peaks are about one order of magnitude smaller than without experimental resolution but the total cross section is of course still the same. These figures suggest that one could be able to measure the $\gamma \gamma \rightarrow \gamma \gamma$ scattering above $W_{\gamma \gamma}>2 \mathrm{GeV}$. Comparing Fig. 8 (a) with (b) one can observe that the relevant distribution varies more than two orders of magnitude. In the case of argon-argon collisions, although the collision energy is larger, the predicted cross section is smaller. This is caused by fourth power of the charge number of the nucleus in the cross section. The photon flux depends on $Z_{A}^{2}$ so the cross section is multiplied by $Z_{A}^{4}$. Thus the total cross section for lead-lead collision is more than two orders of magnitude larger than for the argon-argon collision case.

\section{Conclusion}

The ultraperipheral heavy ion collisions give a possibility to measure the $\gamma \gamma \rightarrow \gamma \gamma$ scattering. So far the ATLAS and CMS collaborations measured the light-by-light scattering for diphoton collisions energies $W_{\gamma \gamma}>6 \mathrm{GeV}$ (ATLAS) and $W_{\gamma \gamma}>5 \mathrm{GeV}$ (CMS). Calculated by us the Standard Model predictions are roughly consistent with the experimental data. Our results include realistic photon fluxes that is a Fourier transform of the charge distribution in the nucleus. We have proposed several additional mechanisms which contribute to two-photon state. Each of them plays a important role and different ranges of diphoton invariant mass or transverse momentum of single photon. Next, we have studied background from two-pion decay into pairs of photons. Here the condition that only two out of four photons in the final state are detected. This contribution could be wrongly interpreted as an enhanced $\gamma \gamma \rightarrow \gamma \gamma$ 
scattering at low energies $\left(M_{\gamma \gamma}<2 \mathrm{GeV}\right)$. The signal to background ratio will be improved by including extra cut i.e. on scalar/vector asymmetry or transverse momentum of the $\gamma \gamma$ pair [23]. The $\gamma \gamma$ scattering through pseudoscalar and scalar mesons $\left(\eta, \eta^{\prime}(958), \eta_{c}(1 S), \eta_{c}(2 S)\right.$, $\left.\chi_{c 0}(1 P)\right)$ have been studied too. The $\gamma \gamma \rightarrow \eta, \eta^{\prime} \rightarrow \gamma \gamma$ resonance scattering could be measured with good statistics. Comparing contributions for lead-lead and argon-argon collisions, one can deduce that collision of lighter nuclei is less favorable. However, we can hope the luminosity in the run with Ar-Ar collision will be higher.

This work has been supported by the Polish National Science Center grant DEC2014/15/B/ST2/02528.

\section{References}

[1] H. Euler and B. Kockel, Naturwiss. 23, 246 (1935)

[2] W. Heisenberg and H. Euler Zeit. f. Phys. 98, 714 (1936)

[3] A. Akhieser, L. Landau and I. Pomeranchuk, Nature 138, 206 (1936)

[4] T. Hahn and M. Perez-Victoria, Comput. Phys. Commun. 118, 153 (1999)

[5] G. J. van Oldenborgh and J. A. M. Vermaseren, Z. Phys. C46, 425 (1990)

[6] M. Kłusek-Gawenda, W. Schäfer and A. Szczurek, Phys. Lett. B761, 399 (2016)

[7] E. Meggiolaro, Phys. Lett. B451, 414 (1999)

[8] P. Lebiedowicz and A. Szczurek, Phys. Lett. B772, 330 (2017)

[9] M. Kłusek-Gawenda and A. Szczurek, Phys. Rev. C87, 054908 (2013)

[10] Particle Data Group (C. Patrignani et al.), Chin. Phys. C40, 100001 (2016)

[11] Belle Collaboration (S. Uehara et al.), Phys. Rev. D79, 052009 (2009)

[12] Crystal Ball Collaboration (H. Marsiske et al.), Phys. Rev. D41, 3324 (1990)

[13] E. Fermi, Nuovo Cimento 2, 143 (1925)

[14] C. F. Weizsäcker, Z. Phys. 88, 612 (1934)

[15] E. J. Williams, Phys. Rev. 45, 729 (1934)

[16] G.P. Lepage, Journal of Computational Physics 27, 192 (1978)

[17] ATLAS Collaboration (M. Aaboud et al.), Nature Phys. 13, 852 (2017)

[18] CMS Collaboration (A. M. Sirunyan et al.), arXiv:1810.04602 [hep-ex]

[19] M. Kłusek-Gawenda, P. Lebiedowicz and A. Szczurek, Phys. Rev. C93, 044907 (2016)

[20] ALICE Collaboration (B. B. Abelev et al.), Int. J. Mod. Phys. A29, 1430044 (2014)

[21] LHCb Collaboration (M. Clemencic et al.), J. Phys. Conf. Ser. 331, 032023 (2011)

[22] F. Bock, CERN-THESIS-2012-444

[23] M. Kłusek-Gawenda, arXiv:1809.03823 [hep-ph] 